\title{
Non-clinical immunogenicity, biodistribution and toxicology evaluation of a chimpanzee adenovirus-based COVID-19 vaccine in rat and rhesus macaque
}

\author{
Xuedong Dai ${ }^{1}$ (1) $\cdot$ Weijun Zhao ${ }^{1} \cdot$ Xin Tong $^{2} \cdot$ Wei Liu $^{1} \cdot$ Xianhuan Zeng $^{1} \cdot$ Xiaohui Duan $^{1} \cdot$ Hua Wu ${ }^{1} \cdot$ Lili Wang $^{2}$. \\ Zhen Huang ${ }^{2} \cdot$ Xinying Tang $^{1} \cdot$ Yong Yang ${ }^{1}$
}

Received: 16 November 2021 / Accepted: 23 December 2021 / Published online: 28 February 2022

(c) The Author(s), under exclusive licence to Springer-Verlag GmbH Germany, part of Springer Nature 2022

\begin{abstract}
Coronavirus Disease 2019 (COVID-19) caused by severe acute respiratory syndrome coronavirus 2 (SARS-CoV-2) emerged in 2019 has rapidly expanded into a serious global pandemic. Due to the high morbidity and mortality of COVID-19, there is an urgent need to develop safe and effective vaccines. AdC68-19S is an investigational chimpanzee adenovirus serotype 68 (AdC68) vector-based vaccine which encodes the full-length spike protein of SARS-CoV-2. Here, we evaluated the immunogenicity, biodistribution and safety profiles of the candidate vaccine AdC68-19S in Sprague Dawley (SD) rat and rhesus macaque under GLP conditions. To characterize the biodistribution profile of AdC68-19S, SD rats were given a single intramuscular injection of AdC68-19S $2 \times 10^{11} \mathrm{VP} /$ dose. Designated organs were collected on day 1, day 2, day 4, day 8 and day 15. Genomic DNA was extracted from all samples and was further quantified by real-time quantitative polymerase chain reaction (qPCR). To characterize the toxicology and immunogenicity profiles of AdC68-19S, the rats and rhesus macaques were injected intramuscularly with AdC68-19S up to $2 \times 10^{11} \mathrm{vp} /$ dose or $4 \times 10^{11} \mathrm{vp} / \mathrm{dose}$ ( 2 and fourfold the proposed clinical dose of $1 \times 10^{11} \mathrm{vp} /$ dose) on two or three occasions with a 14-day interval period, respectively. In addition to the conventional toxicological evaluation indexes, the antigen-specific cellular and humoral responses were evaluated. We proved that multiple intramuscular injections could elicit effective and long-lasting neutralizing antibody responses and Th1 T cell responses. AdC68-19S was mainly distributed in injection sites and no AdC68-19S related toxicological reaction was observed. In conclusion, these results have shown that AdC68-19S could induce an effective immune response with a good safety profile, and is a promising candidate vaccine against COVID- 19.
\end{abstract}

Keywords COVID-19 vaccine · Chimpanzee adenovirus vector · Immunogenicity · Biodistribution · Toxicology

\section{Introduction}

Xuedong Dai, Weijun Zhao and Xin Tong contributed equally to this work.

Zhen Huang

hz@walvax.com

Xinying Tang

1620174466@cpu.edu.cn

Yong Yang

yy@cpu.edu.cn

1 Center for New Drug Safety Evaluation and Research, China Pharmaceutical University, Nanjing, Jiangsu 211198, People's Republic of China

2 Yunnan Walvax Biotech, Co. LTD, Kunming, People's Republic of China
Coronavirus Disease 2019 (COVID-19) caused by SARS$\mathrm{CoV}-2$ infection has rapidly expanded into a serious global pandemic. Most patients infected with SARS-CoV-2 would develop mild to moderate illness. However, some patients would develop acute respiratory distress syndrome and even die. Till now, SARS-CoV-2 has caused more than 200 million infections and 4.3 million deaths worldwide, posing extreme pressure on the global public health and economy. Therefore, vaccines as the most cost-effective way for disease control, is in an urgent demand.

Compared with traditional inactivated vaccines, adenovirus-based vaccines could induce stronger humoral and cellular immune responses at the same time. Since the pandemic, several adenovirus-based vaccines have been authorized for 
emergency use, including CanSino (Ad5) (Zhu et al. 2020), Gamaleya (Ad5 and Ad26) (Logunov et al. 2021), AstraZeneca/Oxford University (ChAdOX1) (Voysey et al. 2021), and Johnson \& Johnson (Ad26) (Sadoff et al. 2021). However, due to pre-existing immunity to the human adenovirus serotype 5 (Ad5), the efficacy of Ad5 vector-based vaccines might be limited. Therefore, SARS-CoV-2 vaccines based on rare adenovirus serotype vectors might exhibit improved efficacy.

AdC68-19S is a recombinant chimpanzee adenovirus serotype 68 (AdC68) vector-based vaccine encoding the full-length spike protein of SARS-CoV-2. AdC68 is a replication-deficient adenovirus vector with complete deletion of $\mathrm{E} 1$ and E3, and there is also partial deletion in E4, which is replaced by the corresponding E4 region of Ad5. Spike protein is one of the structural proteins of SARS-CoV-2 virus. It can recognize receptor and mediate virus entry into host cells and serves as the major target for COVID-19 vaccine (Wang et al. 2020). AdC68-19S has been proved to induce sustained and protective immunity against SARS-CoV-2 infection in BalB/c mice, Golden Syrian Hamster and rhesus macaque (Li et al. 2021). Here, we evaluated the immunogenicity, biodistribution and in vivo toxicological profiles of AdC68-19S in rat and rhesus macaque under good laboratory practice (GLP) conditions. We found that AdC68-19S was mainly distributed at the injection site after intramuscular injection, and no systemic and local toxicity related to AdC68-19S was observed. In conclusion, our results proved that AdC68-19S could induce an effective immune response with a good safety profile, and can be further validated in clinical trials.

\section{Material and methods}

\section{Animal experiments}

The design of preclinical toxicology study was based on good laboratory practice (GLP).

\section{SD rat experiments}

Five to six-week-old SD rats were obtained from SLAC Laboratory Animal Co. Ltd (Shanghai, China) and housed in SPF condition (China Pharmaceutical University). In biodistribution study, two groups of SD rats (15 rats/sex/ group) were injected intramuscularly with AdC68-19S $2 \times 10^{11} \mathrm{vp}$ (twofold the proposed clinical dose of $1 \times 10^{11} \mathrm{vp} /$ dose) or vehicle, respectively, on day 1 . Three rats per sex for each group were bled and euthanized on day $1(\sim 4 \mathrm{~h}$ post injection), day 2, day 4 , day 8 and day 15 . Designated organs including bone marrow, spleen, mesenteric lymph nodes, stomach, colon, kidney, liver, thymus, heart, lung, brain, skeletal muscle, testis $(\widehat{\jmath})$, prostate $(\hat{\jmath})$, ovary $(\phi)$ and uterus ( + ) were collected and frozen. In toxicology study, three groups of 15 male and 15 female SD rats were administered intramuscularly with AdC68-19S $1 \times 10^{11} \mathrm{vp} /$ dose, $2 \times 10^{11} \mathrm{vp} /$ dose ( 1 and twofold the proposed clinical dose of $1 \times 10^{11} \mathrm{vp} /$ dose) or vehicle control, respectively, on day 1 and day 15 . Mortality, morbidity and general health were monitored twice per day. Cage-side observation such as status of behavior, respiratory, circulatory, skin and fur, excretion were evaluated and recorded on a daily basis. Body weights and food consumption were calculated once a week. Rectal temperature was measured 6, 24 and $48 \mathrm{~h}$ after each vaccination. The effect of AdC68-19S on the function of the central nervous system was evaluated by Functional observational battery (FOB) as previously described (Mathiasen and Moser 2018) in three rats per sex per group pre-dose, around 1-2 h, 2 days, 7 days, 13 days post first dose. Ten rats per sex from each group were euthanized 7 days and the remaining animals were sacrificed 21 days after last vaccination. Whole blood and sera for further analysis were collected before euthanization. At scheduled necropsies, histological evaluation was performed on major organs/tissues included adrenal gland, brain, eye, gall bladder, gastino-intestinal tract, kidney, larynx, liver, lungs, mammary gland, mesenteric lymph node, oesophagus, optic nerve, ovary, pancreas, pituitary gland, prostate gland, sciatic nerve, seminal vesicles, skeletal muscle, spinal cord, spleen, sternum, testis, thymus, thyroid, trachea, urinary bladder, uterus and injection sites.

\section{Rhesus macaque experiments}

Three to five-year-old rhesus macaques were purchased from Sheng peng Laboratory Animal Co. Ltd (Anhui, China) and housed in a clean facility (China Pharmaceutical University). Three groups of 5 male and 5 female rhesus macaques were administrated intramuscularly with AdC68-19S $1 \times 10^{11} \mathrm{vp} /$ dose and $4 \times 10^{11} \mathrm{vp} /$ dose ( 1 and fourfold the proposed clinical dose of $1 \times 10^{11} \mathrm{vp} /$ dose) or vehicle, respectively, on day 1, day 15 and day 29. Blood pressure was determined by RM300 Veterinary Multi-parameter Monitor (RWD Life Science, China) at 6, 24 and $48 \mathrm{~h}$ after each vaccination. Electrocardiograph was monitored on surviving animals at 7 and 21 days post the third administration. The RR, PR, QT intervals and heart rate were measured using ECG-9020 K Electrocardiograph (Nihon Kohden, Japan). Corrected QT (QTc) was calculated as follows: $\mathrm{QTc}=\mathrm{QT}-0.087 \times(\mathrm{RR}-$ 1000). Whole blood and sera were collected on day 15 predosing, day 29 pre-dosing, day 36 and day 50 for ELISpot and Neutralization antibody titration. Three animals per sex from each group were euthanized on day 37 and the remaining animals were sacrificed on day 51 . Cage-side observation, body weight and food consumption determination, hematology, clinical chemistry, coagulation and 
histopathological evaluation were conducted as described in SD rat experiments.

\section{Surrogated SARS-Cov-2 virus neutralization test (sVNT)}

The neutralizing antibody titer was determined by sVNT kit (Genscript, China). Briefly, heat-inactivated sera, along with positive and negative controls, were tenfold diluted in sample dilution buffer and the primary screening was conducted according to the manufacture's instruction. All the positive samples were further two-fold serially diluted for titration (SD rats from 1:200 to 1:12,560, rhesus macaques from 1:20 to 1:2560). All samples were tested in duplicate. The neutralization endpoint titer was determined as the reciprocal of the highest serum dilution whose inhibition rate was higher than the cutoff.

\section{Enzyme-linked immunospot (ELISpot) assay}

Peripheral blood lymphocytes (PBMCs) were isolated from $6 \mathrm{~mL}$ freshly collected blood by density gradient centrifuge at room temperature (Fcmacs, China). The PBMCs were counted by Countstar RigelS2 (ALIT Life Science Co. Limited, China) and adjusted to $2.5^{*} 10^{6}$ cells $/ \mathrm{mL}$ in $10 \%$ heat-inactivated fetal bovine serum (Biological Industries, Israel) supplemented RPMI-1640 (Bioind) for further cellular immune response evaluation. Overlapping peptide pools, consisting of 15-mer sequence with 11 amino-acid overlap, covered the complete sequence of SARS-Cov-2 spike protein and were used as antigens. Th1-related IFN- $\gamma$, TNF- $\alpha$, IL-2 and Th2-related IL-4, IL-5, IL-6 ELISpot assay were performed with Monkey IFN- $\gamma / \mathrm{IL}-2 /$ TNF- $\alpha$ FluoroSpot ${ }^{\text {FLEX }}$ Kit and Human IL-4/IL-5/IL-6 FluoroSpot ${ }^{\text {FLEX }}$ Kit (Mabtech, Sweden), respectively, following the instructions. PBMCs were stimulated at the concentration of $1 \mu \mathrm{g} / \mathrm{mL} /$ peptide (day 15) or $2 \mu \mathrm{g} / \mathrm{mL} /$ peptide (day 29, 36 and 50) for around $20 \mathrm{~h}$ for subsequent analysis. Spot forming cells were detected by ImmunoSpot S6 (Cellular Technology, Cleveland, $\mathrm{OH}$ ).

\section{Flow cytometry analysis}

$\mathrm{T}$ cell subset in SD rat or rhesus macaque was evaluated using FACSAria SORP. (BD, San Diego, CA). For SD rats, $100 \mu \mathrm{L}$ whole blood or $10^{6}$ spleenocytes were stained with AF647 anti-rat CD3 (1F4, Biolegend, San Diego, CA), FITC anti-rat CD4 (OX-35, Biolegend), Pe-cy7 anti-rat CD8a (OX-8, eBioscience) and PE anti-rat CD45RC (OX-22, Biolegend). For rhesus macaques, whole blood or spleenocytes were stained with FITC anti-human CD3e (SP34, BD), BV510 anti-human CD4 (OKT4, Biolegend), BV711 anti-human CD8a (RPA-T8, Biolegend), BV421 anti-human
CD197 (G043H7, Biolegend) and PE anti-human CD45RA (MEM-56, Invitrogen). Dead cells were stained with LIVE/ DEAD $^{\text {TM }}$ Fixable Near-IR Dead Cell Stain Kit (Invitrogen). All data were analyzed by Flowjo V10 (Tree Star, Palo Alto, CA).

\section{Hematology, clinical chemistry and coagulation}

Hematological evaluation was performed with EDTA-anticoagulated blood by ADVIA-2120 Hematology Analyzer (Simens, IL). The measured parameters included white blood cell count (WBC), neutrophile granulocyte ratio (\%NEU), lymphocyte ratio (\%LYM), monocyte ratio (\%MONO), eosinophil ratio (\%EOS), basophil ratio (\%BASO), red blood cell count (RBC), hemoglobin (HGB), hematocrit (HCT), mean corpuscular volume (MCV), mean corpuscular hemoglobin (MCH), mean corpuscular-hemoglobin concentration (MCHC), blood platelet count (PLT), reticulocyte percentage (\% Retic) and reticulocyte count (Retic).

Blood samples were taken into tubes containing lithium heparin as an anticoagulant. After centrifugation, the assayed with Dimension Xpand Plus (Simens) for the following parameters: alkaline phosphatase (ALP), alanine aminotransaminase (ALT), aspartate aminotransferase (AST), urea nitrogen (BUN), total protein (TP), albumin (ALB), blood glucose (GLUC), total bilirubin (TBI), creatinine (CREA), total cholesterol (CHOL), triglyceride (TGL) and gamma-glutamyl transferase (GGT).

Blood samples were taken into tubes containing trisodium citrate as anticoagulant and were assayed for: activated partial thromboplastin time (APTT), fibrinogen concentration (FIB), prothrombin time (PT) and thrombin time (TT).

\section{Histopathological evaluation}

Representative samples of the tissues were taken from animals scheduled for necropsy were fixed in 10\% neutral buffered formalin or Davison's fixative (eyeball and testis), and then were dehydrated and embedded in paraffin wax and sectioned at approximately 4-6 $\mu \mathrm{m}$ thickness and stained with hematoxylin and eosin (H\&E) as previously described(Fischer et al. 2008). Microscopic evaluation was performed by an appropriately qualified and experienced veterinary pathologist. Injection site was evaluated by Draize scoring (Vinardell and Mitjans 2008).

\section{Biodistribution study}

A biodistribution study upon single intramuscular administration (SD rats) or repeat intramuscular administrations (rhesus macaques) of AdC68-19S were conducted under GLP condition. The biodistribution assay in rhesus macaque was included in the repeat-dose toxicity study. Three animals 
per sex from high dose $\left(4 \times 10^{11} \mathrm{vp} /\right.$ dose $)$ or vehicle were euthanized 7 days (day 37) after the final immunization while the remaining two animals per sex per group were euthanized 21 days (day 51) post injection. Genomic DNA was extracted from all samples by TIANamp Genomic DNA Kit (Tiangen, China). The adenovirus genomic DNA was further quantified in triplicate using PerfectStart Probe qPCR SuperMix (Transgen, China) with the following primers and probes, hexon forward 5'-ACTGGTTCCTGGTCC AGATG-3', hexon reverse 5'-CGAGTTGTTGTGCTGGTA GG-3' and probe 6-FAM-5'-TCGTCCACCACCTGGCGG CT-3'-6-TAMRA. All data were collected and analyzed in QuantStudio 3 (Thermofisher).

\section{Statistical analysis}

Unless otherwise stated, all statistical tests were two-sided and performed at the 5\% signifance level using SPSS 22.0 (Chicago, IL). If the group variances appeared homogeneous, A one-way ANOVA was used and pairwise comparisons were made using a Dunnett's test only if the ANOVA test was significant. If the variances were heterogeneous, a Kruskal-Wallis non-parametric ANOVA was used and pairwise comparisons were made using a Mann-Whitney test.

\section{Results}

\section{Biodistribution of AdC68-19S genome after single-dose administration in SD rats}

First, we evaluated the biodistribution of AdC68-19S in rats after a single intramuscular injection. Tissues were collected from sacrificed rats on day 1 ( $4 \mathrm{~h}$ post injection), day 2 , day 4 , day 8 and day 15 for genome extraction. Viral genome was determined by qPCR. As exhibited in Fig. 1, AdC68-19S was mainly distributed in muscles (administration location) and the viral genome was around $1 \times 10^{6} \sim 1 \times 10^{7}$ copies per $100 \mu \mathrm{g}$ genomic DNA on day 1. Trace amount of AdC6819S genome could be detected in bone marrow, spleen, mesenteric lymph nodes, stomach, colon, kidney, liver, lung, brain, skeletal muscle, testis and prostate. Since day 2, viral genome in all tissues gradually decreased. Fifteen days after administration, only muscles remained AdC68-19S genomepositive. In conclusion, AdC68-19S mainly existed in the muscle at the injection site and the amount of viral DNA decreased over time. Trace amount of viral DNA can be cleared within two days after injection in most tested tissues.

\section{In vivo immunological response of AdC68-19S in SD rats}

Subsequently, we evaluated the immune response in rats after immunization with AdC68-19S twice. Three groups of SD rats received low dose $\left(1 \times 10^{11} \mathrm{vp} /\right.$ dose $)$, high dose $\left(2 \times 10^{11} \mathrm{vp} / \mathrm{dose}\right)$ of AdC68-19S or vehicle intramuscularly, respectively, on day 1 and day 15 . Neutralizing antibody titration and $\mathrm{T}$ lymphocyte analysis were performed as Fig. 2A. The neutralization endpoint titers of low-dose and high-dose group were higher than those of the control group after boost (day 22) (Fig. 2B). Endpoint neutralizing antibody titers remained at a comparable level on day 36, indicating that AdC68-19S could induce an effective and sustained neutralizing antibody response against SARS$\mathrm{CoV}-2$ in rats. In order to analyze the potential effect of AdC68-19S on rat T cell subsets, we used flow cytometry

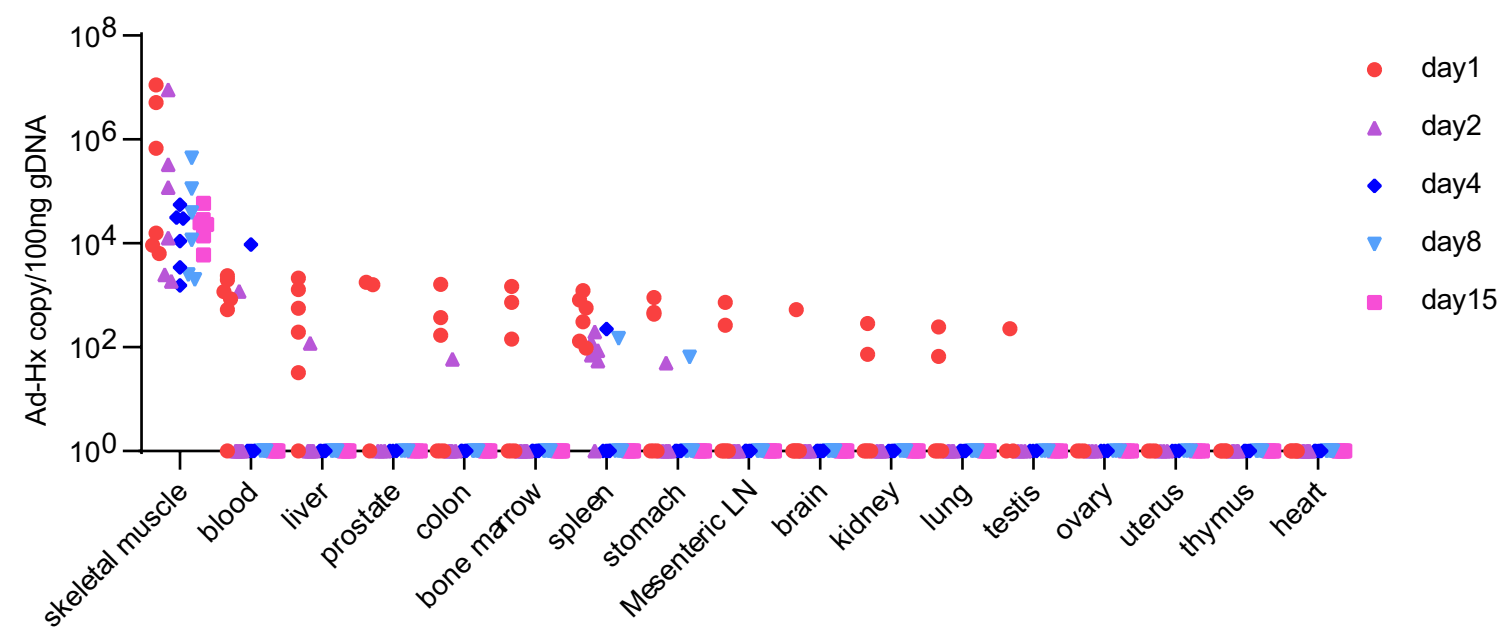

Fig. 1 Biodistribution of AdC68-19S DNA in SD rats after a single intramuscular injection. SD rats were injected intramuscularly with AdC68-19S $2 \times 10^{11} \mathrm{vp}$, three rats per sex for each group were bled and euthanized on day 1 ( $\sim 4$ h post injection), day 2 , day 4 , day 8 and day 15 . Adenovirus genomic DNA was quantified in triplicate by qPCR 
A

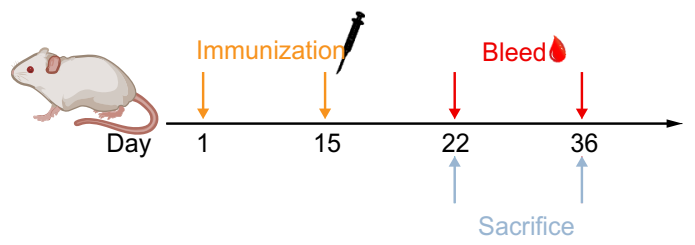

C

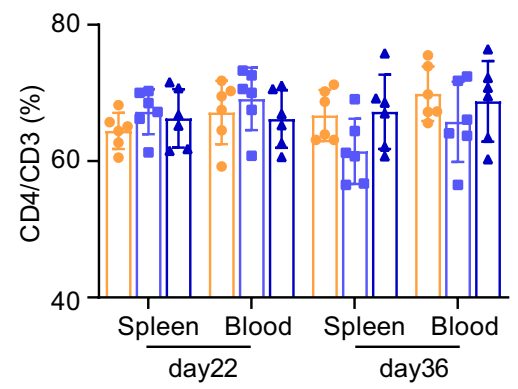

E

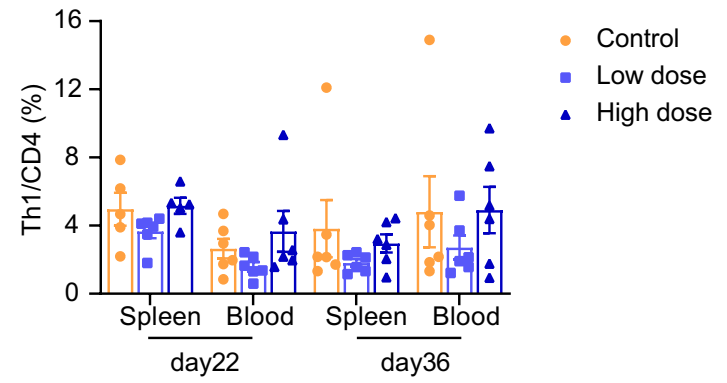

Fig. 2 Immune response of rats immunized with AdC68-19S. A Timeline for immunization and immunological characterization. SD rats were injected intramuscularly with AdC68-19S $1 \times 10^{11} \mathrm{vp} /$ dose (low dose), $2 \times 10^{11} \mathrm{vp} /$ dose (high dose) or vehicle control on day 1 and day 15 , respectively. Three rats per sex from each group were euthanized and biological samples were collected before or after euthanization on day 22 and day 36. B The neutralizing antibody

to determine the proportions of CD4, CD8, Th1 CD4, Th2 CD4 in the peripheral blood and spleen of the animals on day 22 and day 36. As shown in Fig. 2C-F the T cell panel was not significantly changed after immunization compared with the control group.

\section{Toxicology study in SD rats}

To characterize the potential system and local toxicity of AdC68-19S in rat, two groups of SD rats with corresponding controls were examined as described in Material and
B

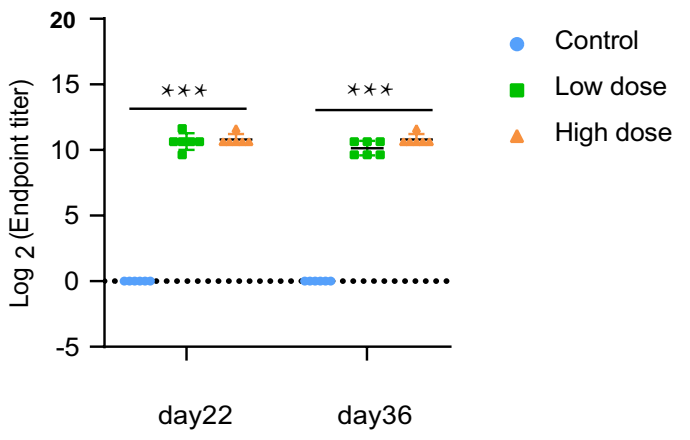

D

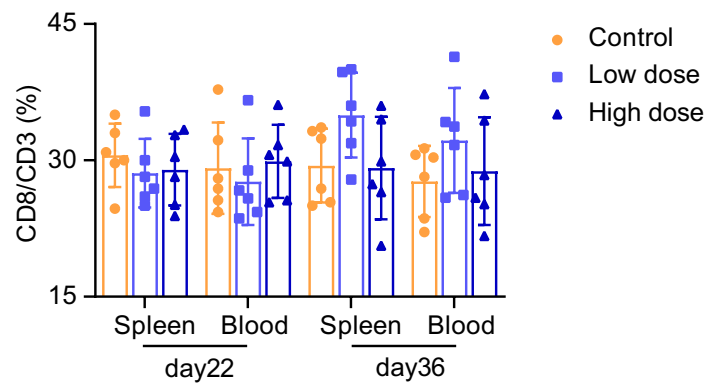

$\mathbf{F}$

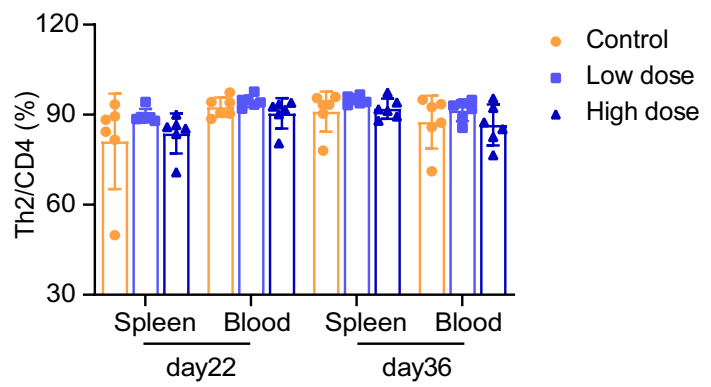

against SARS-CoV-2 of serum samples from rats after immunization was determined by surrogated SARS-Cov-2 virus neutralization test. C-F Proportion of T-lymphocyte subsets of $\mathrm{CD}^{+} \mathrm{T}$ cells. $\mathbf{C ~ C D} 8^{+}$ T cells. D Th1 cells. E and Th2 cells. $\mathbf{F}$ in spleen and blood from immunized rats. Data are presented as means $\pm \mathrm{SD}(n=6)$, ns $=$ no significance, $* p<0.05, * * p<0.01$ and $* * * p<0.001$

Methods. Designated animals were sacrificed to evaluate the potential adverse effects on day 22 and day 36. During the dosing or recovery phase, no AdC68-19S related mortality or clinical signs were observed. Body weights were recorded once a week as presented in Fig. 3A. No relevant effects on body weight were found in any vaccine group. Rectal temperature remained consistent within $48 \mathrm{~h}$ after vaccination (Fig. 3B). In addition, there were no organ weight changes in vaccine groups (Fig. 3C). There were no AdC68-19S related hematology changes in any vaccine group on day 22 and day 36 (Table 1). Compared with the control group, there was no 
A

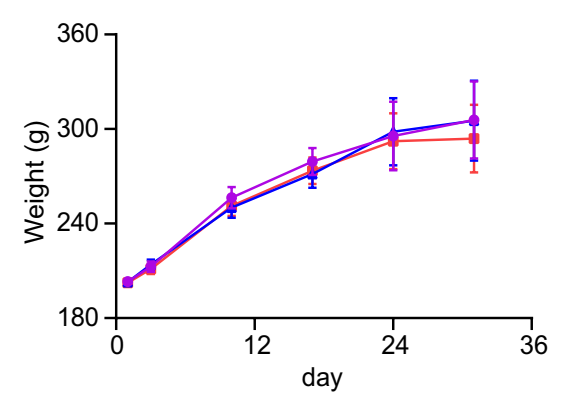

C
B

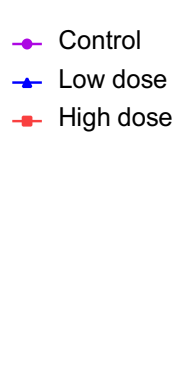

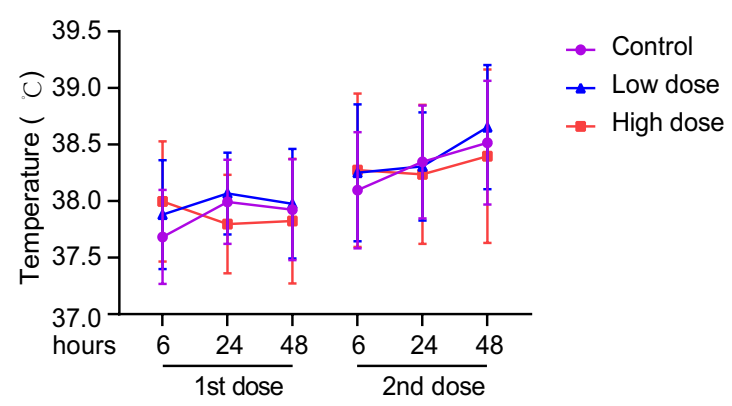

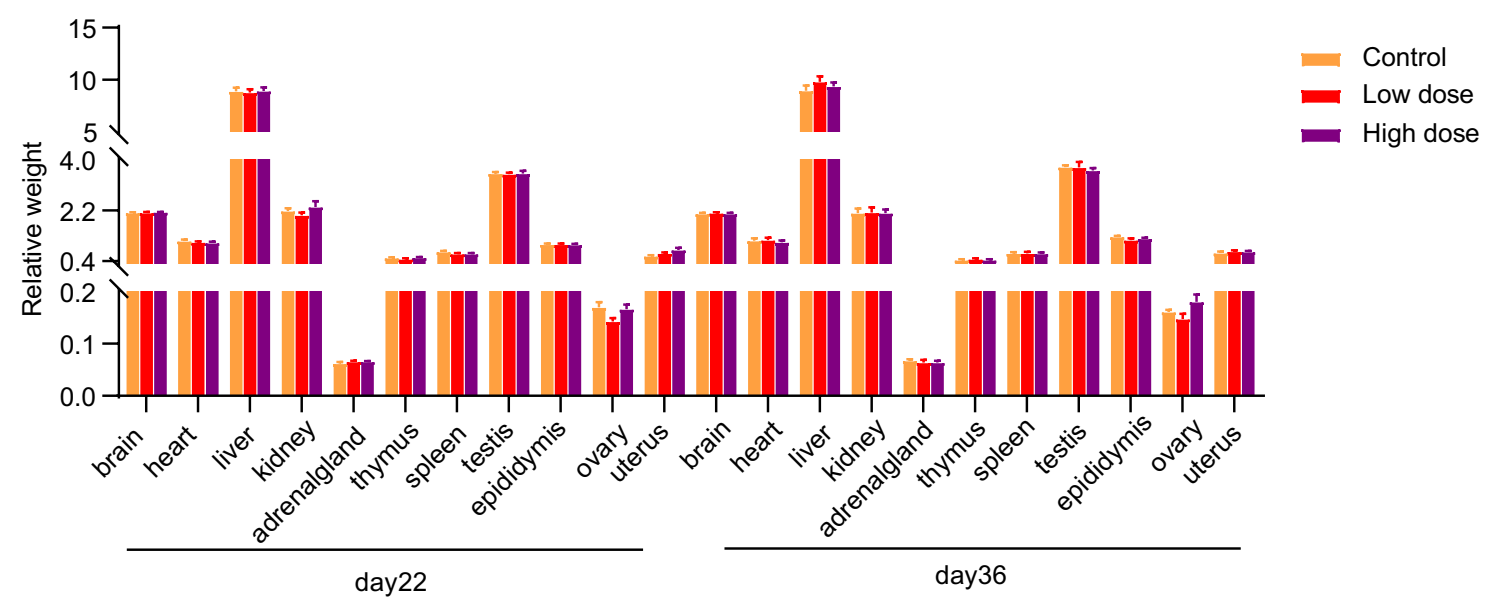

D

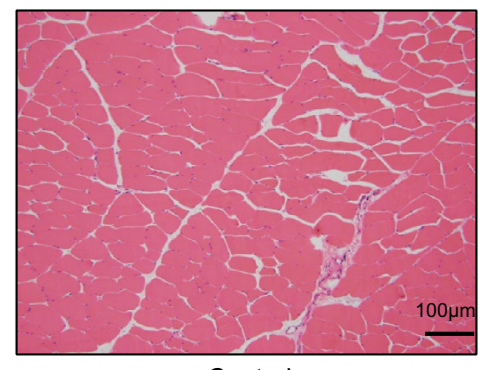

Control

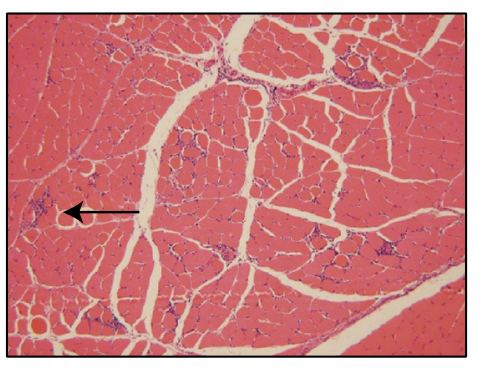

Low dose

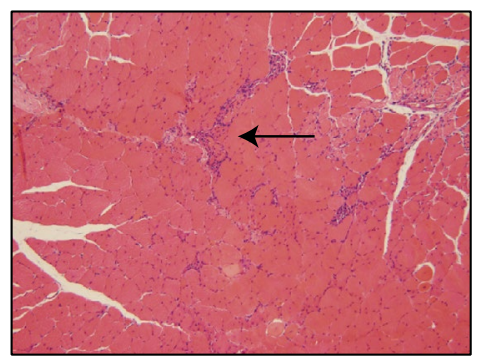

High dose
Fig. 3 Toxicity of AdC68-19S in rats. SD rats were injected intramuscularly with AdC68-19S $1 \times 10^{11} \mathrm{vp} /$ dose (low dose), $2 \times 10^{11} \mathrm{vp} /$ dose (high dose) or vehicle control on day 1 and day 15 , respectively. Ten and five rats per sex from each group were euthanized and necropsied on day 22 and day 36, respectively. A The body weight of rats during the dosing and recovery phase. $\mathbf{B}$ The rectal tempera- ture at the $6 \mathrm{~h}, 24 \mathrm{~h}$ and $48 \mathrm{~h}$ after each vaccination in rats. C Organ weight of rats at scheduled necropsies. D H\&E staining of representative sections of muscle tissue at the injection site of rats injected with AdC68-19S $(\mathrm{Bar}=100 \mu \mathrm{m})$. The arrows indicate sites of inflammation significant difference in other clinical chemistry parameters on day 22 and day 36 except that TP in the high dose group increased slightly on day 22 (Table 2). The slight increase of TP may be related to the increase of antibody level induced by vaccine. Minimal and transient prolongation of APTT and TT in low and high dose groups was seen On day 22 $(P<0.05)$ (Table 3). Statistically significant differences of APTT and TT between control and vaccinated animals were consistent with normal variation and considered incidental. Due to the trace distribution of AdC68-19S DNA in rat brain, FOB was performed to evaluate the potential effect on the central nervous system. The results showed that one intramuscular dose of AdC68-19S up to $2 \times 10^{11} \mathrm{vp} / \mathrm{dose}$ (twofold the proposed clinical dose) had no obvious effect on the central nervous system in rats (Data not shown). 


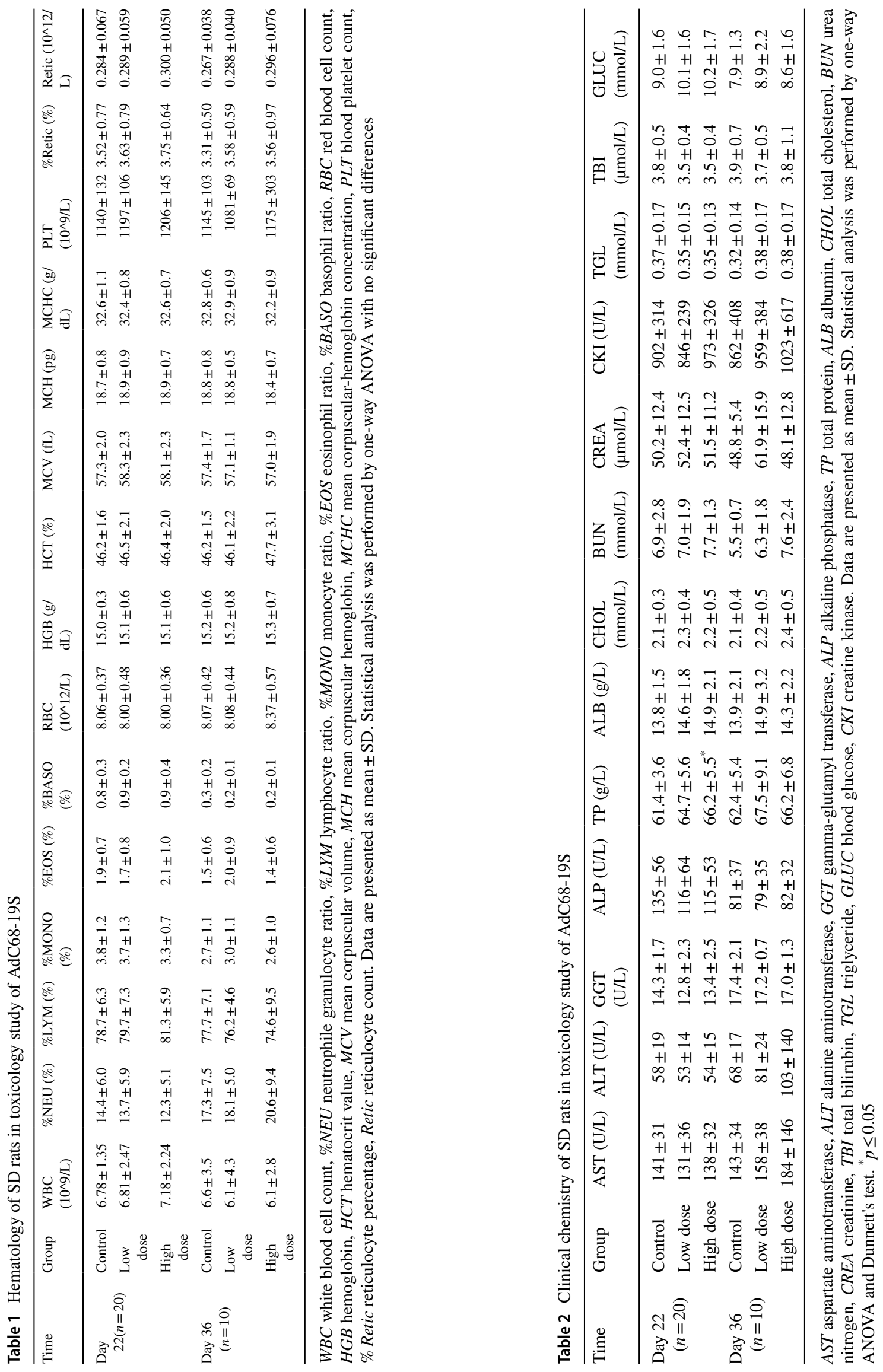


Table 3 Coagulation of SD rats in toxicology study of AdC68$19 \mathrm{~S}$

\begin{tabular}{llllll}
\hline Time & Group & PT(s) & APTT(s) & TT(s) & FIB $(\mathrm{g} / \mathrm{L})$ \\
\hline Day 22 $(n=20)$ & Control & $17.0 \pm 1.1$ & $22.6 \pm 2.9$ & $31.4 \pm 3.6$ & $1.72 \pm 0.23$ \\
& Low dose & $17.1 \pm 0.9$ & $25.0 \pm 2.1^{*}$ & $33.8 \pm 2.8^{*}$ & $1.68 \pm 0.29$ \\
& High dose & $16.7 \pm 0.8$ & $25.7 \pm 1.9^{*}$ & $33.9 \pm 2.1^{*}$ & $1.75 \pm 0.27$ \\
Day 36 $(n=10)$ & Control & $16.9 \pm 1.1$ & $23.0 \pm 3.5$ & $32.0 \pm 1.7$ & $1.90 \pm 0.21$ \\
& Low dose & $16.7 \pm 1.1$ & $24.3 \pm 2.8$ & $30.6 \pm 2.7$ & $2.02 \pm 0.29$ \\
& High dose & $16.9 \pm 1.6$ & $26.7 \pm 5.0$ & $31.2 \pm 4.6$ & $2.00 \pm 0.41$ \\
\hline
\end{tabular}

$P T$ prothrombin time, $A P T T$ activated partial thromboplastin time, $T T$ thrombin time, FIB fibrinogen concentration. Data are presented as mean \pm SD. Statistical analysis was performed by one-way ANOVA and Dunnett's test. ${ }^{*} P \leq 0.05$
We evaluated the local effect of AdC68-19S on the muscle at the injection site by Draize scoring. No AdC68-19S related injection site redness, swelling, necrosis or ulcers were noted in all animals. Microscopic evaluation of fixed H\&E-stained paraffin sections was performed by a veterinary pathologist on specified tissues including the skeletal muscle at the injection sites. At the terminal necropsy (day 22), mild interstitial inflammation was observed in the skeletal muscle at the injection sites of animals injected with AdC68-19S, and the incidence was significantly higher than that of the control group (Fig. 3D). At the recovery necropsy (day 36), interstitial inflammation in the skeletal muscle was observed at a lower incidence, demonstrating the reversibility of the microscopic findings. Low incidence of muscle fiber degeneration and/or necrosis was observed in all groups, which was considered to be related to mechanical injury due to the injection procedure because the severity and incidence were consistent among groups. All other sporadic microscopic findings observed at the terminal and recovery necropsies were representative of spontaneously arising background pathology in SD rats of this age and on this kind of study. In conclusion, no toxicology target organs were found in rat, AdC68-19S only induced mild and reversible interstitial inflammation in muscle at the injection site which was considered as non-adverse effect and related to immune response subsequent to vaccination.

\section{In vivo immunological response of AdC68-19S in rhesus macaques}

To better mimic the clinical response, we further evaluated the immunological response and toxicity in rhesus macaques. Rhesus macaques were injected intramuscularly with AdC68-19S $1 \times 10^{11} \mathrm{vp} /$ dose and $4 \times 10^{11} \mathrm{vp} /$ dose $(1$ and fourfold the proposed clinical dose) on day 1, 15 and 29, and immunological responses evaluation were performed as shown in Fig. 4A. The neutralizing antibody could be detected in some animals as early as the 15th day after the first immunization. The neutralizing antibody titer significantly raised after the second dose (day 29) in vaccination groups and gradually increased after the second boost (day 36). Endpoint neutralizing antibody titer remained at a comparable level on day 50, indicating that AdC68-19S could induce a sustained humoral immune response against SARSCOV-2 in macaques (Fig. 4B). ELISpot analysis showed that spike-specific T cells secreting IFN- $\gamma$, TNF- $\alpha$, and IL-2 were detected in PBMC from vaccinated rhesus macaques after stimulation with antigen peptide pools, indicating the Th1biased immune response (Fig. 4 C-F). We further analyze the potential effect of AdC68-19S on T cell subsets by flow cytometry, we found that $\mathrm{T}$ cell panel was not significantly changed after immunization compared with the control group (Fig. 5).

\section{Biodistribution and toxicology study in rhesus macaques}

The biodistribution profiles of AdC68-19S in rhesus macaques were analyzed by qPCR on day 37 and day 51 after three intramuscular injections with an interval of two weeks. Similar to biodistribution in SD rats, AdC68-19S DNA mainly existed in the skeletal muscle at the injection site. AdC68-19S genome could also be detected in bone marrow, spleen, stomach, colon in individual macaques (Table 4). Considering the biodistribution analysis were performed at the 7 days or 21 days after the third administration, AdC68-19S genome copy was relatively low in all positive samples.

We further assessed the potential adverse effects in rhesus macaques as described in Material and Methods. There were no AdC68-19S-related clinical signs during the dosing or recovery phase. Body weights were recorded once a week and no apparent AdC68-19S-related effects were noted (Fig. 6A). There were no obvious abnormalities related to AdC68-19S in body temperature (Fig. 6B), relative organ weight (Fig. 6C) and mean arterial pressure (Fig. 6D) after each administration. The ECG morphology and indicators (including RR, PR, QT, QTc intervals and heart rate) showed no abnormal changes related to AdC68-19S on day36 and day 50 (Fig. 6E). Hematology, clinical chemistry and coagulation 
A

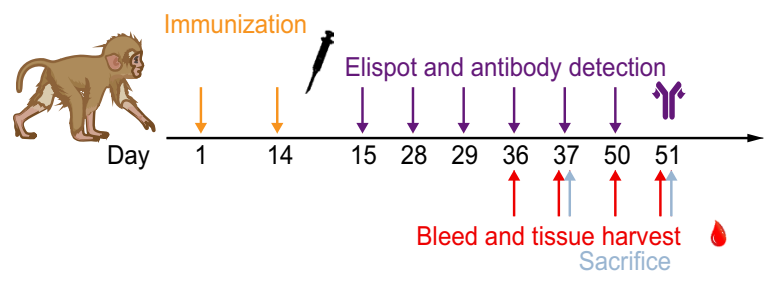

C

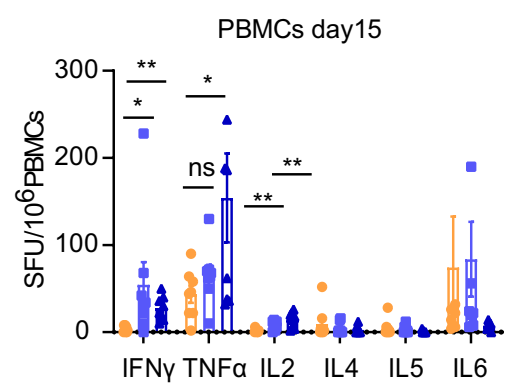

E

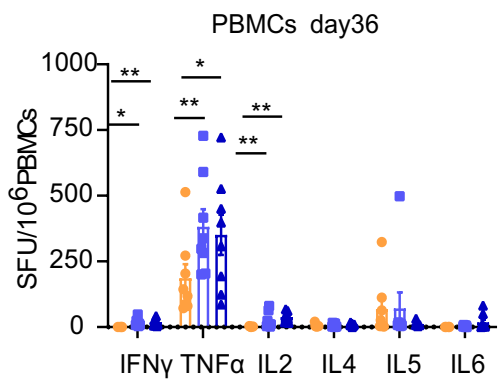

Fig. 4 Immune response of rhesus macaques immunized with AdC68-19S. A Timeline for immunization and immunological characterization. Rhesus macaques were injected intramuscularly with AdC68-19S $1 \times 10^{11} \mathrm{vp} /$ dose (low dose), $4 \times 10^{11} \mathrm{vp} /$ dose (high dose) or vehicle control three times with an interval of 2 weeks. Blood samples were collected from rhesus macaques on day $15(n=8)$, day $29(\mathrm{n}=8)$, day $36(n=8)$ and day $50(n=4)$ for further test. B The neutralizing antibody against SARS-CoV-2 of serum samples

evaluation were performed on day 0 (pre-dosing), day 36 and day 50 (Table 5, 6 and 7). No AdC68-19S related adverse effects on clinical pathology parameters were noted. Statistically significant differences observed in the clinical pathology parameters were consistent with normal biologic variation and were not attributed to AdC68-19S because these differences were minor, lacked dosage-relationship or reflected trends present before vaccination commenced. These included minimally higher RBC in the low and high-dose group on day $36(P<0.05)$, minimally higher BUN in low-dose group on day $36(P<0.05)$, minimally higher ALT in high-dose group and BUN in low-dose group on day $50(P<0.05)$, minimally lower TP in low-dose group on day $50(P<0.05)$. Compared with the control group, there was no effect of vaccination on
B

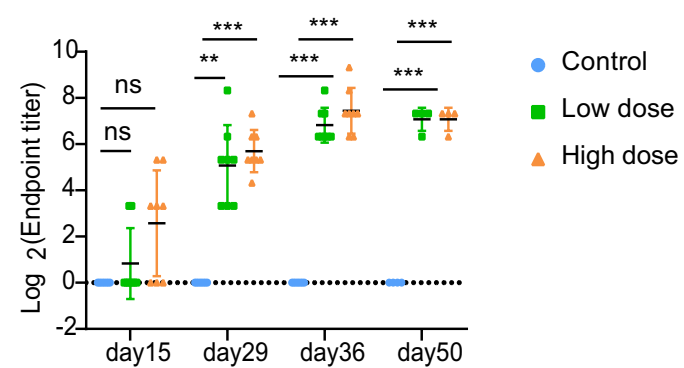

D

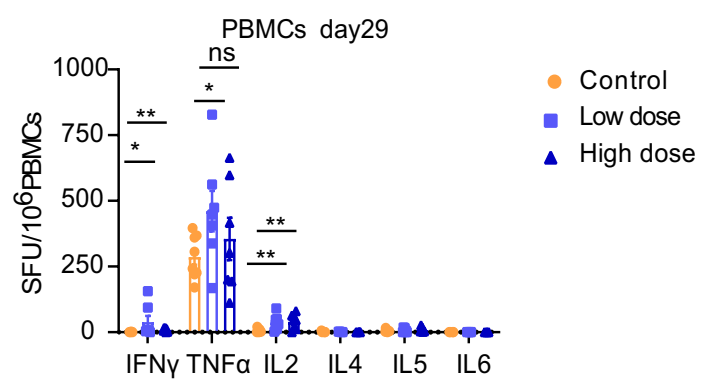

$\mathbf{F}$

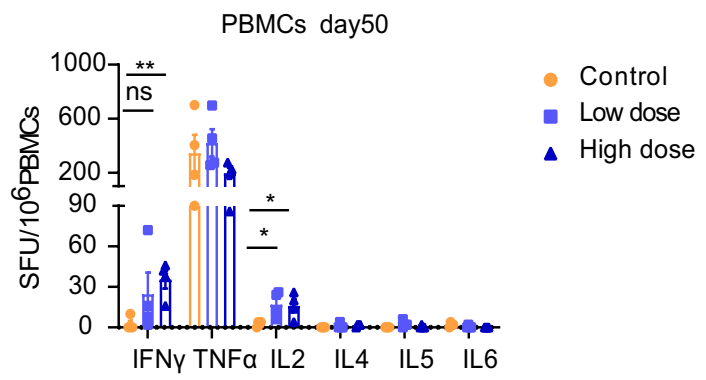

from rhesus macaques after immunization was determined by surrogated SARS-Cov-2 virus neutralization test. C-F. ELISpot analysis of Th1-related IFN- $\gamma$, TNF- $\alpha$, IL-2 and Th2-related IL-4, IL-5, IL-6 positive PBMCs collected from rhesus macaques on day 15 , day 29 , day 36 and day 50 using overlapping peptide pools of spike protein as antigens. Data are presented as mean \pm SD. ns $=$ no significance, $* p<0.05, * * p<0.01$ and $* * * p<0.001$

coagulation on day 36 and day 50. Animals designated for terminal or recovery necropsy (3 and 2 animals/sex/group, respectively) were anesthetized and necropsied on day 37 and day51. Draize scoring was used to evaluate the local toxicity of AdC68-19S on the muscles at the injection site. No AdC6819S related injection site redness, swelling, necrosis or ulcers were noted. Microscopic evaluation of fixed H\&E-stained paraffin sections was performed by a veterinary pathologist on specified tissues including the skeletal muscle at the injection sites. Similar with the pathological results in rat toxicology study, dose dependent minimal to mild interstitial inflammation was observed in the skeletal muscles at the injection sites of animals designated for terminal or recovery necropsy in vaccination groups, and considered reversible according to the 
A
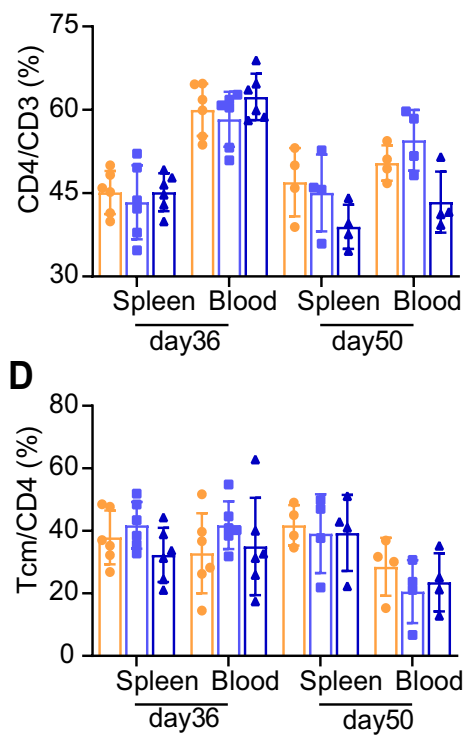

$\mathbf{F}$

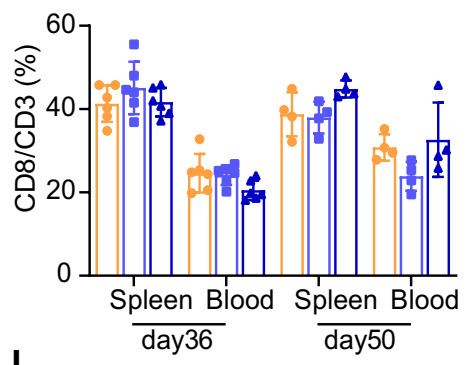

I

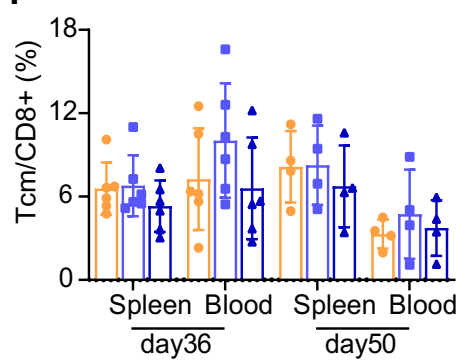

B

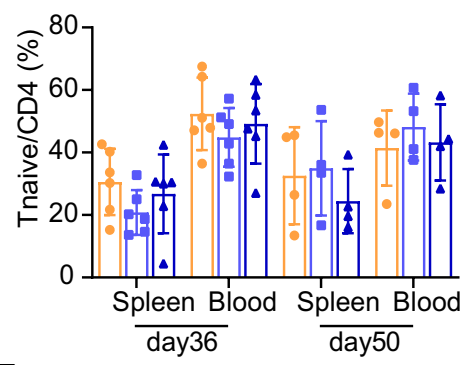

E

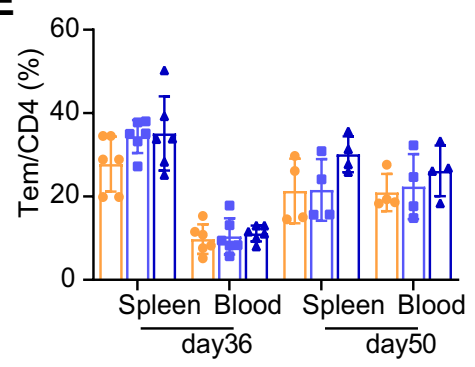

G
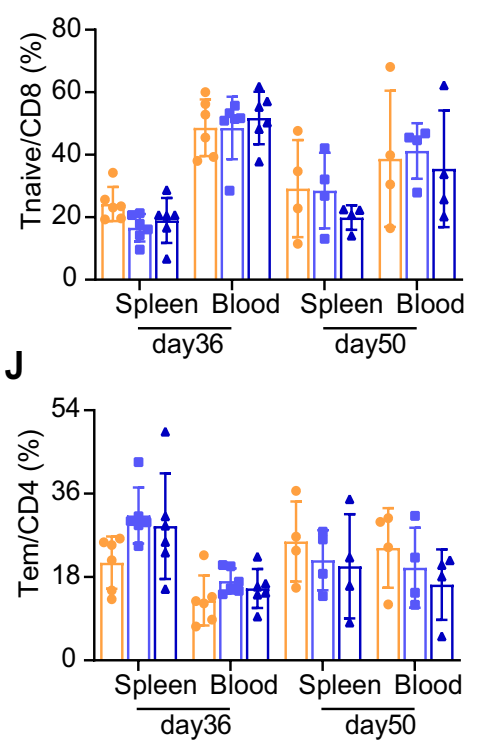

C

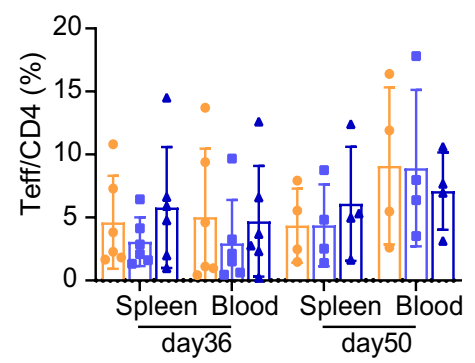

H

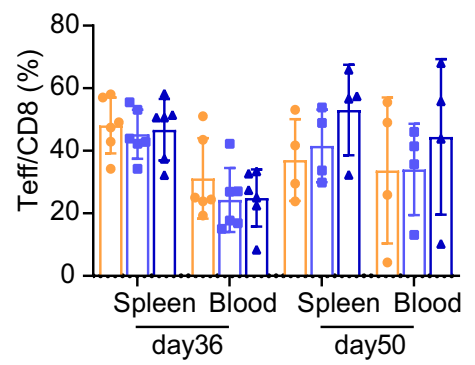

- Control

- Low dose

- High dose
Fig. 5 Proportion of T-lymphocyte subsets in spleen and blood from rhesus macaques. Rhesus macaques were injected intramuscularly with AdC68-19S $1 \times 10^{11} \mathrm{vp} /$ dose (low dose), $4 \times 10^{11} \mathrm{vp} /$ dose (high dose) or vehicle control three times with an interval of two weeks. Blood and splenocytes were collected from rhesus macaques scheduled for necropsy on day $36(n=6)$ and day $50(n=4)$. The frequency of $\mathrm{CD} 4+\mathrm{T}$ cells $(\mathbf{A})$, naïve $\mathrm{CD} 4+\mathrm{T}$ cells $(\mathbf{B})$, effector $\mathrm{CD} 4+\mathrm{T}$ cells $(\mathbf{C})$, central memory $\mathrm{CD} 4+\mathrm{T}$ cells $(\mathbf{D})$, effector memory CD4 + T cells $(\mathbf{E}), \mathrm{CD} 8+\mathrm{T}$ cells $(\mathbf{F})$, naïve CD8 + T cells $(\mathbf{G})$, effector $\mathrm{CD} 8+\mathrm{T}$ cells $(\mathbf{H})$, central memory $\mathrm{CD} 8+\mathrm{T}$ cells $(\mathbf{I})$ and effector memory $\mathrm{CD}^{+} \mathrm{T}$ cells was evaluated by flow cytometry method. Data are presented as mean $\pm \mathrm{SD}$. ns $=$ no significance, $* p<0.05$, $* * p<0.01$ and $* * * p<0.001$ incidence and degree of inflammation (Fig. 6F). Apart from reversible inflammation at the injection site, neither AdC6819S related systemic pathological changes nor local irritation were observed in vaccinated animals, indicating a good safety profile of AdC68-19S in rhesus macaques.

\section{Discussion}

We evaluated the immunogenicity and safety profiles of AdC68-19S in SD rat and rhesus macaque in accordance with GLP to support proceeding to first in human (FIH) 


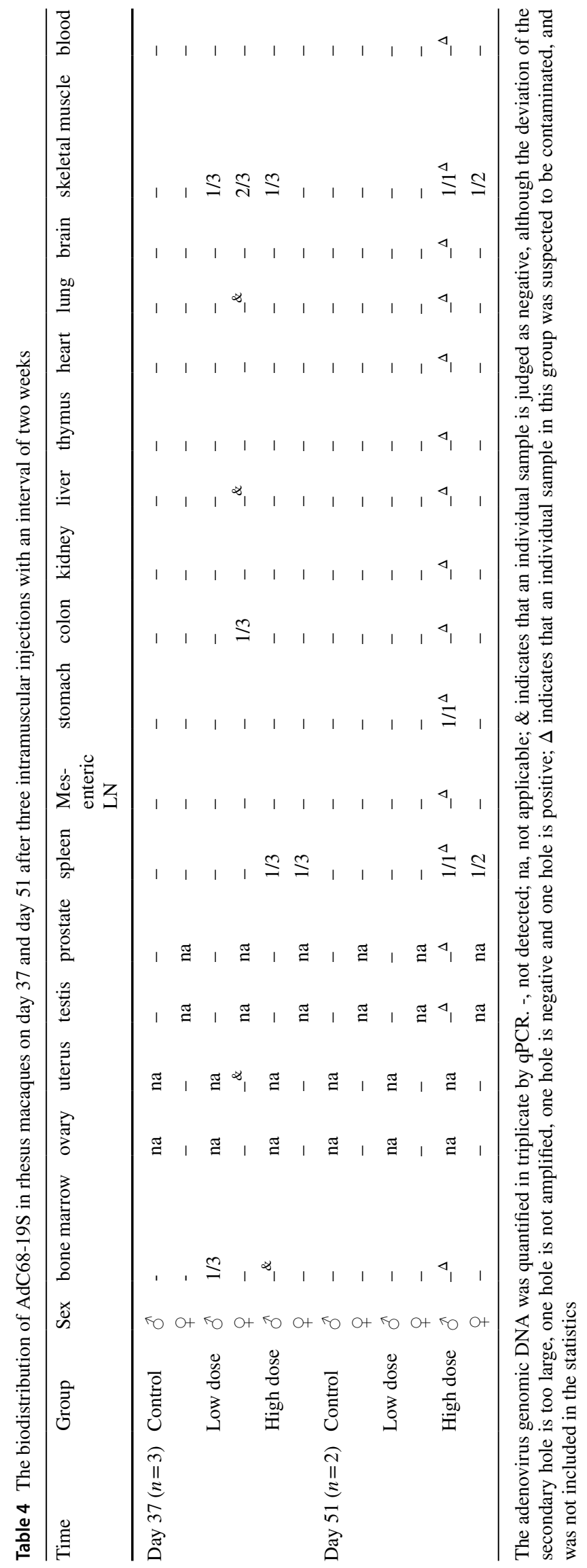


A

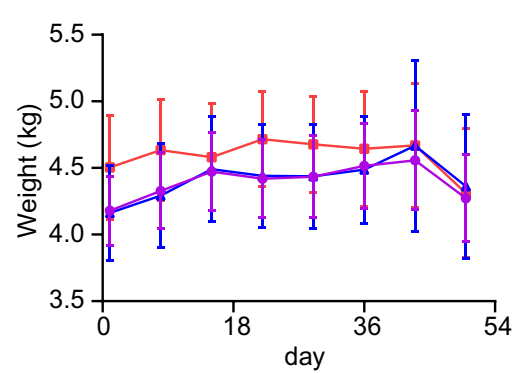

C

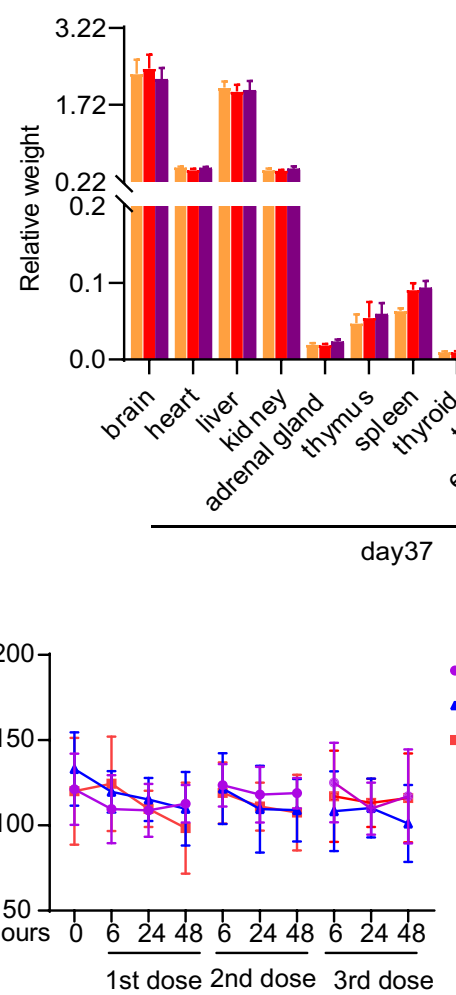

$\mathbf{F}$

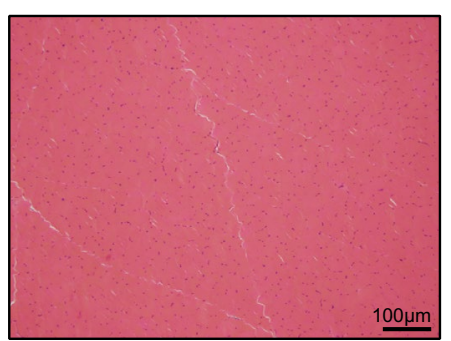

Control

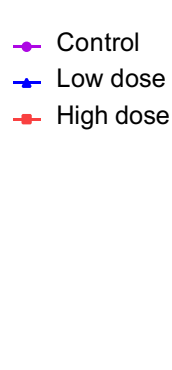

B

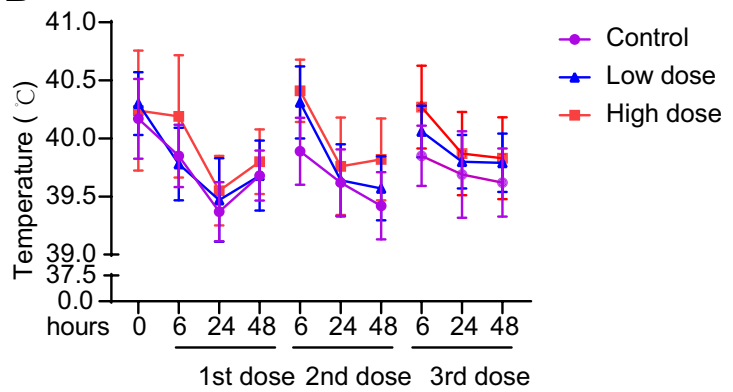

1st dose 2 nd dose 3 rd dose

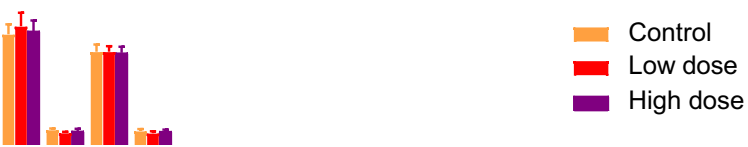

High dose

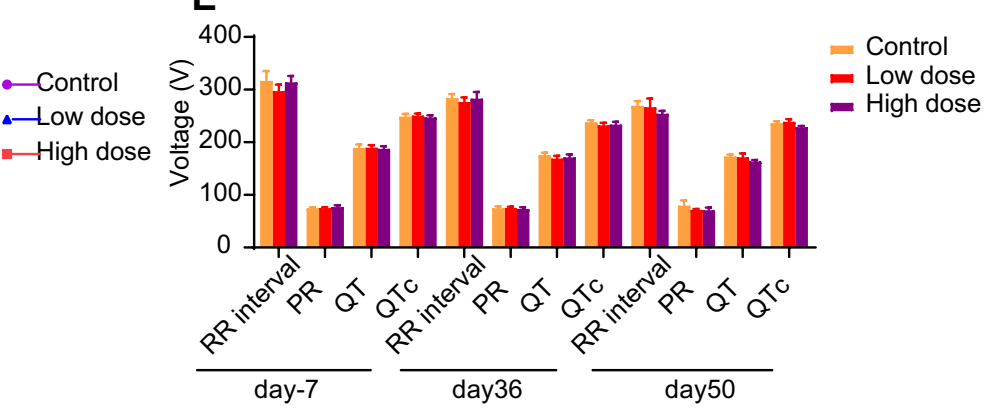

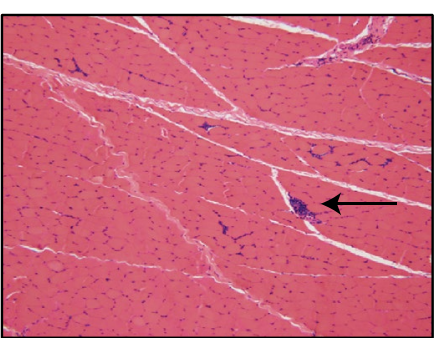

Low dose

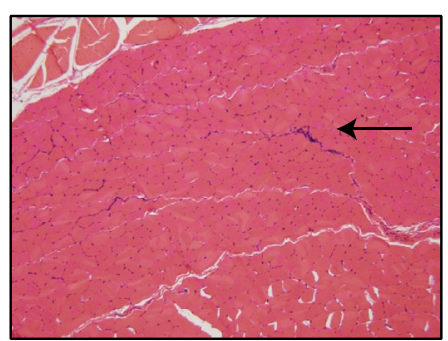

High dose
Fig. 6 Toxicity of AdC68-19S in rhesus macaques. Rhesus macaques were injected intramuscularly with AdC68-19S $1 \times 10^{11} \mathrm{vp} /$ dose (low dose), $4 \times 10^{11} \mathrm{vp} /$ dose (high dose) or vehicle control three times with an interval of 2 weeks. Three and two rhesus macaques per sex from each group were euthanized and necropsied on day 37 and day 51, respectively. A The body weight of rhesus macaques during the dosing and recovery phase. B The rectal temperature at the $6 \mathrm{~h}, 24 \mathrm{~h}$ and $48 \mathrm{~h}$ after each vaccination in rhesus macaques. C Organ weight of rhesus macaques at scheduled necropsies. D Changes mean arterial pressure at different days after each vaccination in rhesus macaques $(n=10)$. E Electrocardiogram of rhesus macaques before and after vaccination on day $-7(n=10)$, day $36(n=10)$ and day $50(n=4)$. Data are presented as mean \pm SD. F H\&E staining of representative sections of muscle tissue at the injection site of rats injected with AdC68-19S $(\mathrm{Bar}=100 \mu \mathrm{m})$. The arrows indicate sites of inflammation 


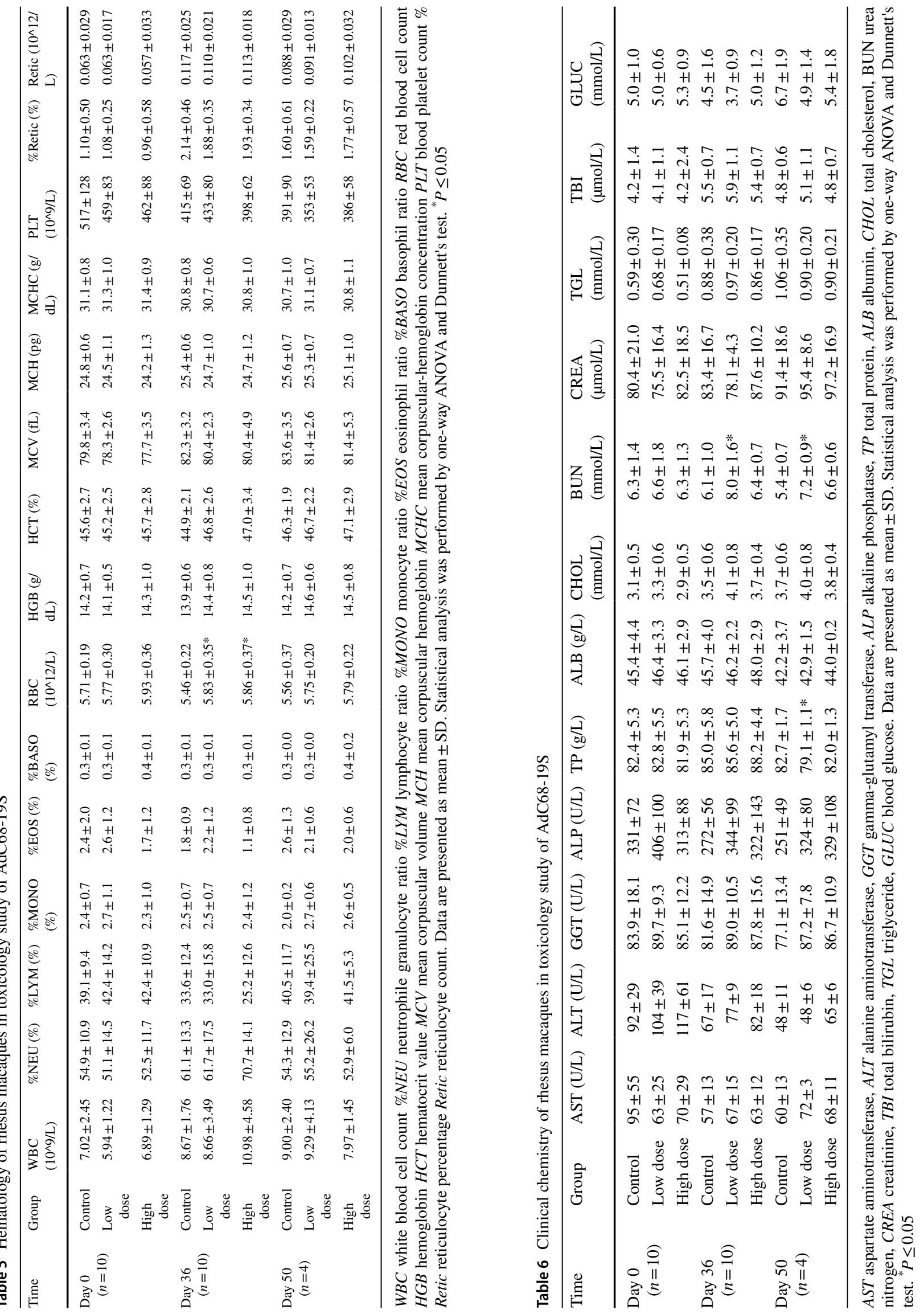


Table 7 Coagulation of rhesus macaques in toxicology study of AdC68-19S

\begin{tabular}{llllll}
\hline Time & Group & PT(s) & APTT(s) & TT(s) & FIB(g/L) \\
\hline Day 0 $(n=10)$ & Control & $13.1 \pm 0.9$ & $22.9 \pm 2.2$ & $12.0 \pm 0.7$ & $2.39 \pm 0.36$ \\
& Low dose & $13.2 \pm 1.0$ & $22.4 \pm 0.8$ & $12.1 \pm 0.8$ & $2.29 \pm 0.37$ \\
& High dose & $13.6 \pm 1.0$ & $23.0 \pm 1.5$ & $12.3 \pm 0.8$ & $2.26 \pm 0.17$ \\
Day 36 $(n=10)$ & Control & $12.8 \pm 0.7$ & $22.6 \pm 0.9$ & $12.1 \pm 0.5$ & $2.42 \pm 0.44$ \\
& Low dose & $12.5 \pm 0.7$ & $22.3 \pm 1.2$ & $11.6 \pm 0.7$ & $2.46 \pm 0.30$ \\
& High dose & $12.9 \pm 0.7$ & $22.7 \pm 1.4$ & $11.9 \pm 0.3$ & $2.28 \pm 0.24$ \\
Day 50 $(n=4)$ & Control & $12.4 \pm 0.3$ & $23.5 \pm 1.5$ & $12.6 \pm 0.7$ & $2.38 \pm 0.36$ \\
& Low dose & $13.0 \pm 0.9$ & $23.1 \pm 1.8$ & $13.1 \pm 0.9$ & $2.23 \pm 0.34$ \\
& High dose & $12.4 \pm 0.7$ & $24.2 \pm 2.6$ & $12.6 \pm 0.4$ & $2.32 \pm 0.16$ \\
\hline
\end{tabular}

$P T$ prothrombin time, APTT activated partial thromboplastin time, TT thrombin time, FIB fibrinogen concentration. Data are presented as mean \pm SD. Statistical analysis was performed by one-way ANOVA with no significant differences clinical trials. All studies were designed in accordance with relevant guidelines on the nonclinical evaluation of vaccines issued by regulatory authorities (WHO Technical Report Series no. 927, 2005; EMA CHMP/VWP/141697/2009). The rat and rhesus macaque were standard test species for use in toxicity studies and previous report has proved that AdC68-19S can induce humoral and cellular immune responses in BalB/c mice, Golden Syrian Hamster and rhesus macaque (Li et al. 2021). The rats and rhesus macaques were injected intramuscularly with AdC68-19S up to $2 \times 10^{11} \mathrm{vp} /$ dose or $4 \times 10^{11} \mathrm{vp} /$ dose on two or three occasions with a 14-day interval period respectively. The highest dose in rat and rhesus macaque was 2- and fourfold more than the proposed clinical dose on a vp/dose basis, and the number of vaccinations of the test animals exceeded the single dose intended for humans. Which are accepted by regulatory authorities. A sustained neutralizing antibody in rat and rhesus macaque and Th1 $\mathrm{T}$ cell response in rhesus macaques to SARS-Cov-2 spike protein contained in the vector were elicited, which qualifies the species for the safety assessment of the AdC68-19S. As for the toxicological responses, AdC6819S only induced mild and reversible interstitial inflammation of muscle at the injection site which was considered non adverse and related to the local inflammation subsequent to vaccination, no other AdC68-19S related unexpected effects were observed. In summary, we proved that AdC68-19S is a safe and effective COVID-19 candidate vaccine for further clinical trials.

Although the vector AdC68 used in AdC68-19S is a replication-deficient adenovirus vector with complete deletion of E1 and E3, indicating that no new infectious virus particles can be produced in the body after vaccination. However, it may be distributed from the injection site to other parts of the body and biodistribution of the vaccine should be studied in order to identify potential toxicological target organs. After a single intramuscular injection, AdC68-19S DNA mainly existed in the muscle at the injection sites of rats and the number of viral DNA copies gradually decreased over time. Trace amount of viral DNA was transiently detected in other tested tissues or organs including brain. Similar to biodistribution in SD rats, AdC68-19S DNA mainly existed in the skeletal muscle at the injection site after three intramuscular injections in rhesus macaque.

In general, these results are consistent with previous studies on chimpanzee adenovirus 3 vector (ChAd3)-based vaccine ChAd3-EBO-Z (Planty et al. 2020), Ad5- and Ad35vectored vaccines injected intramuscularly to rats or rabbits (Sheets et al. 2008). It is worth noting that the receptors used by these viruses to gain entry into cells are different, Ad35 predominately binds CD46 whereas Ad5, ChAd3 and AdC68 binds coxackie adenovirus receptor (Cohen et al. 2002; Gaggar et al. 2003). Since the biodistribution data was determined by the measurement of viral DNA, it was not possible to distinguish between infectious virus particles and degraded adenoviruses. We hypothesize that the cells transduced by the adenovectors at the injection site traffic the viral DNA to specific organs, not adenovectors themselves distributed to other tissues directly. This hypothesis is consistent with the results of toxicology studies in rat and rhesus macaque, that neither AdC68-19S related systemic pathological changes nor local irritation were observed in vaccinated animals except for mild and reversible interstitial inflammation in muscle at the injection site. Interstitial inflammation in muscle at the injection site is a common reaction of vaccines, which was considered as a non-adverse effect and related to immune response subsequent to vaccination (Herve et al. 2019).

Safety is the primary goal of vaccination for healthy people. Vaccination may make the subsequent SARS-CoV-2 infection more serious. Vaccine associated enhanced diseases (VAED) refers to the aggravation of clinical symptoms after pathogen infection in individuals who have received a prior vaccination. Vaccine-associated enhanced respiratory disease (VAERD) is a kind of VAED, which refers to the 
aggravation of lower respiratory tract symptoms of vaccinated people after encountering pathogen infection. Several vaccine-related diseases with clear evidence in history are related to respiratory virus and are caused by inactivated vaccine injection (Graham 2020; Haynes et al. 2020; Munoz et al. 2021; Zellweger et al. 2020). VAERD was reported in infants in the clinical trials of fully inactivated viral vaccines against measles and respiratory syncytial virus (RSV) after clinical infection due to natural exposure in the 1960s (Acosta et al. 2015; Fulginiti et al. 1967; Jorquera et al. 2016; Kim et al. 1969; Vinardell and Mitjans 2008). The main reason for VAERD is considered to be the induction of antibodies with low affinity and little to no neutralizing activity. During natural infection, a large number of antigenantibody complexes were formed to activate complement and cause inflammation and tissue damage. There were a large number of Th2 CD4 T cells and eosinophils in lung tissue (Polack et al. 2003; Ruckwardt et al. 2019). In preclinical studies of Middle East respiratory syndrome (MERS)-CoV and SARS-CoV-1 vaccines from different vaccine platforms, immunopathological evidence of Th2 type hypersensitivity similar to VAERD was observed in the lungs of animal models (Agrawal et al. 2016; Arvin et al. 2020; Yasui et al. 2008). In the development of COVID-19 vaccine, previous work on RSV vaccine should be considered to avoid or reduce the potential risk of VAERD. A promising vaccine should be able to induce high-quality neutralizing antibodies and avoid inducing $\mathrm{Th} 2$ biased immune responses. In our study, an effective and sustained neutralizing antibody response against SARS-COV-2 was induced in rat and rhesus macaque, and Th1 skewing of immune response confirmed in rhesus macaque. AdC68-19S has been proved to induce protective immunity against SARS-CoV-2 infection and reduced lung pathology in previous challenge studies in Golden Syrian Hamster and rhesus macaque ( $\mathrm{Li}$ et al. 2021). Although there is limited awareness and understanding of the value of existing animal models in predicting the potential risk of VAERD in humans of COVID-19 vaccines, the results from the previous study in combination with our present study indicate that the theoretical risk that AdC68$19 \mathrm{~S}$ would predispose for VAERD is minimal.

Since March 2021, rare blood clotting disorder cases known as thrombosis with thrombocytopenia syndrome (TTS) have been reported after vaccinations with ChAdOx1S (AstraZeneca/Oxford) and Ad26.COV2-S (Johnson \& Johnson Janssen) COVID-19 vaccines, which resulted in a temporary pause in vaccination these products in Europe and US (Cines and Bussel 2021; Greinacher et al. 2021; Muir et al. 2021). After case evaluation by national and international agencies, it was concluded that there was a reasonable causal relationship between the two adenovirus-based vaccines and the events. Although the reports of transient thrombocytopenia after intravenous adenovirus injection are consistent in different animals and humans (Othman et al. 2007), there are few reports under the route of intramuscular injection, this may be related to the lower level of adenovirus exposure in the blood after intramuscular administration. The clinical process of TTS has common characteristics with autoimmune heparin-induced thrombocytopenia (aHIT), another rare blood clotting disorders (Greinacher et al. 2017), and is often associated with the positive detection of anti-platelet factor 4 (PF4) antibodies. However, TTS after vaccination does not result from previous exposure to heparin (Cines and Bussel 2021; Scully et al. 2021). Although little is known so far, the pathophysiology of TTS is thought to be similar to that of aHIT's. The anti-PF4 antibodies bound to the complex of PF4-polyanion (unknown or yet to be characterized) subsequently binds to FcR $\gamma$ IIA receptor on the platelets, resulting in platelet activation and aggregation, which leads to platelet consumption, thrombocytopenia and thrombosis (Alam 2021). Although evidence of the pathological antibody role of PF4 is being established, it is still necessary to clarify which component of the vaccine can form a complex with PF4, and whether genetic factors may play a role in the immune response leading to thrombocytopenia after vaccination. At the time of writing, the exact pathophysiological mechanisms of TTS are still unclear, a continued study into this issue is critical. In our study, there were no AdC68-19S related changes in platelet count and related hematology parameters in rat and rhesus macaque, as well as related clinical signs and histopathological changes, which were in line with the toxicity study results of ChAdOx1-S and Ad26.COV2-S COVID-19 vaccines disclosed by regulatory agencies. However, these data are not enough to identify the potential risk of TTS of this chimpanzee adenovirus-based vaccine due to the limited number of animals and differences of immune system between animal and human. However, it should be emphasized that TTS following COVID-19 vaccination remains a rare issue, and the benefits of COVID-19 vaccination far outweigh its potential TTS risks, which has also been unanimously recognized by most regulatory agencies following a thorough safety review. WHO reviewed the existing information and issued an interim emergency guidance to raise awareness of TTS and help doctors assess and manage the potential TTS cases. In future clinical trials, we will closely monitor the vaccinated subjects. If clinical symptoms are suspected to be related to TTS, we will timely carry out laboratory and imaging diagnosis according to the recommendations of WHO guidance and give appropriate treatment quickly to ensure the safety of the subjects.

In conclusion, after single or repeated intramuscular injections, AdC68-19S mainly distributed in the skeletal muscle at the injection site, could elicit antigen-specific neutralizing antibody and Th1 T cell response in rat and rhesus macaque. AdC68-19S was well tolerated in rat and rhesus 
monkey, with only mild and reversible interstitial inflammation of muscle at the injection site, no obvious systemic and local toxicity was observed. Based on these data, AdC68$19 \mathrm{~S}$ is considered to be a promising COVID-19 candidate vaccine and suitable for further validation in human clinical trials. In addition, since the biodistribution and safety of recombinant viral vectored vaccine appear more dependent on vector platform than on its encoded antigen, these data can be used to support the clinical development of other AdC68-based candidate vaccines. Avoiding repetition of studies can not only greatly save time and resources, but also accelerate the development of vaccine.

Author contributions All the authors contributed to the idea and design of this study. XT, LW and ZH sponsored the study. The test operation, data collection and statistical analysis were carried out by XD, WZ, WL, XZ, XD, HW, XT. The first draft of the manuscript was written by XD, WZ, XT, XT and all authors reviewed and commented on the draft of the manuscript. ZH, XT and YY approved the final manuscript. $\mathrm{XD}, \mathrm{WZ}, \mathrm{XT}$ contribute equally to this work.

Funding This work was funded by Yunnan Walvax Biotech, Co. LTD.

\section{Declarations}

Conflicts of interest The authors declare that they have no conflict of interest.

Compliance with ethical standards All animal experiment protocols were reviewed and approved by the Institutional Animal Care and Use Committee of Center for New Drug Safety Evaluation and Research, China Pharmaceutical University.

\section{References}

Acosta PL, Caballero MT, Polack FP (2015) Brief history and characterization of enhanced respiratory syncytial virus disease. Clin Vaccine Immunol 23(3):189-195. https://doi.org/10.1128/CVI. 00609-15

Agrawal AS, Tao X, Algaissi A, Garron T, Narayanan K, Peng BH, Couch RB, Tseng CT (2016) Immunization with inactivated Middle East Respiratory Syndrome coronavirus vaccine leads to lung immunopathology on challenge with live virus. Hum Vaccin Immunother 12(9):2351-2356. https://doi.org/10.1080/21645515. 2016.1177688

Alam W (2021) COVID-19 vaccine-induced immune thrombotic thrombocytopenia: a review of the potential mechanisms and proposed management. Sci Prog 104(2):1-13. https://doi.org/10. 1177/00368504211025927

Arvin AM, Fink K, Schmid MA, Cathcart A, Spreafico R, HavenarDaughton C, Lanzavecchia A, Corti D, Virgin HW (2020) A perspective on potential antibody-dependent enhancement of SARS-CoV-2. Nature 584(7821):353-363. https://doi.org/10. $1038 / \mathrm{s} 41586-020-2538-8$

Cines DB, Bussel JB (2021) SARS-CoV-2 vaccine-induced immune thrombotic thrombocytopenia. N Engl J Med 384(23):2254-2256. https://doi.org/10.1056/NEJMe2106315
Cohen CJ, Xiang ZQ, Gao GP, Ertl H, Wilson JM, Bergelson JM (2002) Chimpanzee adenovirus CV-68 adapted as a gene delivery vector interacts with the coxsackievirus and adenovirus receptor. J Gen Virol 83(Pt 1):151-155. https://doi.org/10.1099/ 0022-1317-83-1-151

EMA (2010) CHMP/VWP/141697/2009 Guideline on quality, nonclinical and clinical aspects of live recombinant viral vectored vaccine. https://www.ema.europa.eu/en/quality-non-clinical-clini cal-aspects-live-recombinant-viral-vectored-vaccines. Accessed 7 Nov 2021

Fischer AH, Jacobson KA, Rose J, Zeller R (2008) Hematoxylin and eosin staining of tissue and cell sections. CSH Protoc 2008:t4986. https://doi.org/10.1101/pdb.prot4986

Fulginiti VA, Eller JJ, Downie AW, Kempe CH (1967) Altered reactivity to measles virus. Atypical measles in children previously immunized with inactivated measles virus vaccines. JAMA 202(12):1075-1080. https://doi.org/10.1001/jama.202.12.1075

Gaggar A, Shayakhmetov DM, Lieber A (2003) CD46 is a cellular receptor for group B adenoviruses. Nat Med 9(11):1408-1412. https://doi.org/10.1038/nm952

Graham BS (2020) Rapid COVID-19 vaccine development. Science 368(6494):945-946. https://doi.org/10.1126/science.abb8923

Greinacher A, Selleng K, Warkentin TE (2017) Autoimmune heparininduced thrombocytopenia. J Thromb Haemost 15(11):20992114. https://doi.org/10.1111/jth.13813

Greinacher A, Thiele T, Warkentin TE, Weisser K, Kyrle PA, Eichinger S (2021) Thrombotic Thrombocytopenia after ChAdOx1 nCov-19 Vaccination. N Engl J Med 384(22):2092-2101. https://doi.org/ 10.1056/NEJMoa2104840

Haynes BF, Corey L, Fernandes P, Gilbert PB, Hotez PJ, Rao S, Santos MR, Schuitemaker H, Watson M, Arvin A (2020) Prospects for a safe COVID-19 vaccine. Sci Transl Med. https://doi.org/10.1126/ scitranslmed.abe0948

Herve C, Laupeze B, Del GG, Didierlaurent AM, Tavares DSF (2019) The how's and what's of vaccine reactogenicity. Npj Vaccines 4:39. https://doi.org/10.1038/s41541-019-0132-6

Jorquera PA, Anderson L, Tripp RA (2016) Understanding respiratory syncytial virus (RSV) vaccine development and aspects of disease pathogenesis. Expert Rev Vaccines 15(2):173-187. https://doi.org/ 10.1586/14760584.2016.1115353

Kim HW, Canchola JG, Brandt CD, Pyles G, Chanock RM, Jensen K, Parrott RH (1969) Respiratory syncytial virus disease in infants despite prior administration of antigenic inactivated vaccine. Am J Epidemiol 89(4):422-434. https://doi.org/10.1093/oxfordjour nals.aje.a120955

Li M, Guo J, Lu S, Zhou R, Shi H, Shi X, Cheng L, Liang Q, Liu H, Wang P et al (2021) Single-dose immunization with a chimpanzee adenovirus-based vaccine induces sustained and protective immunity against SARS-CoV-2 infection. Front Immunol 12:697074. https://doi.org/10.3389/fimmu.2021.697074

Logunov DY, Dolzhikova IV, Shcheblyakov DV, Tukhvatulin AI, Zubkova OV, Dzharullaeva AS, Kovyrshina AV, Lubenets NL, Grousova DM, Erokhova AS et al (2021) Safety and efficacy of an rAd26 and rAd5 vector-based heterologous prime-boost COVID19 vaccine: an interim analysis of a randomised controlled phase 3 trial in Russia. Lancet 397(10275):671-681. https://doi.org/10. 1016/S0140-6736(21)00234-8

Mathiasen JR, Moser VC (2018) The Irwin test and functional observational battery (FOB) for assessing the effects of compounds on behavior, physiology, and safety pharmacology in rodents. Curr Protoc Pharmacol 83(1):e43. https://doi.org/10.1002/cpph.43

Muir KL, Kallam A, Koepsell SA, Gundabolu K (2021) Thrombotic thrombocytopenia after Ad26.COV2.S vaccination. N Engl J Med 384(20):1964-1965. https://doi.org/10.1056/NEJMc2105869

Munoz FM, Cramer JP, Dekker CL, Dudley MZ, Graham BS, Gurwith M, Law B, Perlman S, Polack FP, Spergel JM et al (2021) 
Vaccine-associated enhanced disease: case definition and guidelines for data collection, analysis, and presentation of immunization safety data. Vaccine 39(22):3053-3066. https://doi.org/10. 1016/j.vaccine.2021.01.055

Othman M, Labelle A, Mazzetti I, Elbatarny HS, Lillicrap D (2007) Adenovirus-induced thrombocytopenia: the role of von Willebrand factor and P-selectin in mediating accelerated platelet clearance. Blood 109(7):2832-2839. https://doi.org/10.1182/ blood-2006-06-032524

Planty C, Chevalier G, Duclos ME, Chalmey C, Thirion-Delalande C, Sobry C, Steff AM, Destexhe E (2020) Nonclinical safety assessment of repeated administration and biodistribution of ChAd3EBO-Z Ebola candidate vaccine. J Appl Toxicol 40(6):748-762. https://doi.org/10.1002/jat.3941

Polack FP, Hoffman SJ, Crujeiras G, Griffin DE (2003) A role for nonprotective complement-fixing antibodies with low avidity for measles virus in atypical measles. Nat Med 9(9):1209-1213. https://doi.org/10.1038/nm918

Ruckwardt TJ, Morabito KM, Graham BS (2019) Immunological lessons from respiratory syncytial virus vaccine development. Immunity 51(3):429-442. https://doi.org/10.1016/j.immuni.2019. 08.007

Sadoff J, Gray G, Vandebosch A, Cardenas V, Shukarev G, Grinsztejn B, Goepfert PA, Truyers C, Fennema H, Spiessens B et al (2021) Safety and efficacy of single-dose Ad26.COV2.S vaccine against Covid-19. N Engl J Med 384(23):2187-2201. https://doi.org/10. 1056/NEJMoa2101544

Scully M, Singh D, Lown R, Poles A, Solomon T, Levi M, Goldblatt D, Kotoucek P, Thomas W, Lester W (2021) Pathologic antibodies to platelet factor 4 after ChAdOx1 nCoV-19 vaccination. $\mathrm{N}$ Engl J Med 384(23):2202-2211. https://doi.org/10.1056/NEJMo a2105385

Sheets RL, Stein J, Bailer RT, Koup RA, Andrews C, Nason M, He B, Koo E, Trotter H, Duffy C et al (2008) Biodistribution and toxicological safety of adenovirus type 5 and type 35 vectored vaccines against human immunodeficiency virus-1 (HIV-1), Ebola, or Marburg are similar despite differing adenovirus serotype vector, manufacturer's construct, or gene inserts. J Immunotoxicol 5(3):315-335. https://doi.org/10.1080/15376510802312464
Vinardell MP, Mitjans M (2008) Alternative methods for eye and skin irritation tests: an overview. J Pharm Sci 97(1):46-59. https://doi. org/10.1002/jps.21088

Voysey M, Clemens S, Madhi SA, Weckx LY, Folegatti PM, Aley PK, Angus B, Baillie VL, Barnabas SL, Bhorat QE et al (2021) Safety and efficacy of the ChAdOx1 nCoV-19 vaccine (AZD1222) against SARS-CoV-2: an interim analysis of four randomised controlled trials in Brazil, South Africa, and the UK. Lancet 397(10269):99-111. https://doi.org/10.1016/S0140-6736(20) 32661-1

Wang Q, Zhang Y, Wu L, Niu S, Song C, Zhang Z, Lu G, Qiao C, Hu Y, Yuen KY et al (2020) Structural and functional basis of SARSCoV-2 entry by using human ACE2. Cell 181(4):894-904. https:// doi.org/10.1016/j.cell.2020.03.045

WHO (2005) Technical Report Series no. 927 Annex 1-WHO guidelines on non clinical evaluation of vaccines. https://www.who. int/publications/m/item/annex1-nonclinical.p31-63. Accessed 7 Nov. 2021

Yasui F, Kai C, Kitabatake M, Inoue S, Yoneda M, Yokochi S, Kase R, Sekiguchi S, Morita K, Hishima T et al (2008) Prior immunization with severe acute respiratory syndrome (SARS)-associated coronavirus (SARS-CoV) nucleocapsid protein causes severe pneumonia in mice infected with SARS-CoV. J Immunol 181(9):6337-6348. https://doi.org/10.4049/jimmunol.181.9.6337

Zellweger RM, Wartel TA, Marks F, Song M, Kim JH (2020) Vaccination against SARS-CoV-2 and disease enhancement-knowns and unknowns. Expert Rev Vaccines 19(8):691-698. https://doi.org/ 10.1080/14760584.2020.1800463

Zhu FC, Guan XH, Li YH, Huang JY, Jiang T, Hou LH, Li JX, Yang BF, Wang L, Wang WJ et al (2020) Immunogenicity and safety of a recombinant adenovirus type-5-vectored COVID-19 vaccine in healthy adults aged 18 years or older: a randomised, double-blind, placebo-controlled, phase 2 trial. Lancet 396(10249):479-488. https://doi.org/10.1016/S0140-6736(20)31605-6

Publisher's Note Springer Nature remains neutral with regard to jurisdictional claims in published maps and institutional affiliations. 